\title{
NHE5 regulates growth factor signaling, integrin trafficking, and degradation in glioma cells
}

\author{
Toru Kurata $^{1} \cdot$ Vinotheni Rajendran $^{2} \cdot$ Steven Fan $^{2} \cdot$ Tetsuo Ohta $^{1} \cdot$ Masayuki Numata ${ }^{2} \cdot$ Sachio Fushida $^{1} \mathbb{C}$
}

Received: 3 May 2019 / Accepted: 30 September 2019 / Published online: 8 October 2019

(C) The Author(s) 2019

\begin{abstract}
$\mathrm{Na}^{+} / \mathrm{H}^{+}$exchanger 5 (NHE5) is enriched in neurons and cycles between recycling endosomes and plasma membranes and transports protons to the endosomal lumen as well as to the extracellular space. Although NHE5 expression is undetectable in normal astrocytes, C6 glioma cells express NHE5 at an elevated level. Using C6 cells as a model, here we demonstrate that NHE5 has an important role in tumor growth and tumor cell proliferation and invasion. Glioma xenografts originating from NHE5-knockdown cells exhibited significantly slower growth than those from NHE1-knockdown cells and control cells. Histological characterization of the migration front of $N H E 5$-knockdown tumors revealed a less invasive and less proliferative appearance than NHE1-knockdown and control tumors. NHE5-knockdown but not NHE1-knockdown led to downregulation of fetal bovine serum (FBS)-induced MET and EGFR signaling. Moreover, depletion of NHE5 but not NHE1 reduced the ability of cells to spread on collagen. We found that NHE5 depletion greatly abrogated endocytic recycling and the protein stability of $\beta 1$-integrin, which in part accounted for the defective cell adhesion, spreading, and invasion of NHE5-knockdown cells.
\end{abstract}

Keywords $\beta 1$-integrin $\cdot$ C6 glioma $\cdot$ EGFR $\cdot$ MET $\cdot$ NHE5

$\begin{array}{ll}\text { Abbreviations } \\ \text { NHE } & \mathrm{Na}^{+} / \mathrm{H}^{+} \text {exchanger } \\ \text { FBS } & \text { Fetal bovine serum } \\ \text { ECM } & \text { Extra cellular matrix } \\ \text { EGFR } & \text { Epidermal growth factor receptor } \\ \text { HGF } & \text { Hepatocyte growth factor } \\ \text { hITGB1 } & \text { Human integrin } \beta 1 \\ \text { RIPA } & \text { Radioimmunoprecipitation assay } \\ \text { SDS } & \text { Sodium dodecyl sulfate } \\ \text { PAGE } & \text { Poly-acrylamide gel electrophoresis } \\ \text { APH } & \text { Acid phosphate assay } \\ \text { PFA } & \text { Paraformaldehyde } \\ \text { PEI } & \text { Polyethyleneimine }\end{array}$

Toru Kurata, Vinotheni Rajendran and Steven Fan have contributed equally to this work.

Sachio Fushida

fushida@staff.kanazawa-u.ac.jp

1 Department of Gastroenterological Surgery, Kanazawa University Graduate School of Medical Science, 13-1

Takara-machi, Kanazawa 920-8641, Japan

2 Department of Biochemistry and Molecular Biology, University of British Columbia, Vancouver, BC V6T 1Z3, Canada
PBS Phosphate buffered saline

HA Human influenza hemagglutinin

DMEM Dulbecco's modified Eagle's medium

NKA Sodium/potassium-ATPase

TRKA Tropomyosin receptor kinase A

\section{Background}

Acidic extracellular $\mathrm{pH}$ is a hallmark of the tumor microenvironment, where proton-pumping activity in malignant tumor cells has a critical role in establishing extracellular acidity by exporting protons produced by tumor metabolism [1]. $\mathrm{Na}^{+} / \mathrm{H}^{+}$exchanger NHE1 is one of the most extensively studied $\mathrm{pH}$-regulating ion transporters [2,3]. Since the discovery of NHE1 as a growth factor-activatable electroneutral antiporter that secretes protons from the cytosol to the extracellular space [4], 12 additional NHE genes have been identified in the human genome [5, 6]. Among the different NHE isoforms, NHE1 has received the most attention as a potential target for anti-cancer therapy. Although previous studies showed the importance of NHE1 in cell proliferation [7-10], little is known about the involvement of other NHE isoforms that may be expressed in malignant tumor cells. 
NHE5 was originally identified as NHE whose mRNA $[11,12]$ and protein expression [13] were detected in the brain, particularly in neuron-enriched brain regions. More recently, NHE5 protein was shown to be abundantly expressed in C6 glioma cells while NHE5 being undetectable in normal astrocytes [14]. NHE5 cycles between recycling endosomes and the plasma membrane [15], and acidifies the lumen of recycling endosomes [16], thereby playing a critical role in regulating the cell-surface availability of the high-affinity nerve growth factor receptor TrkA in PC12 pheochromocytoma cells of adrenal gland origin [17] and the hepatocyte growth factor/scatter factor (HGF) receptor MET in C6 glioma cells [14]. In the current study, we have investigated the involvement of NHE5 and NHE1 in glioma cell signaling, proliferation, and tumor growth through characterization of C6 glioma-based knockdown cell lines.

\section{Materials and methods}

\section{Molecular biology}

Plasmid-based short hairpin RNA (shRNA) constructs against NHE1, NHE5, and scrambled control (N5shA, $\mathrm{N} 5 \mathrm{shB}$, and $\mathrm{N} 5 \mathrm{shC}$, respectively), and C6 cell lines stably expressing shRNA were described previously [14]. Unless otherwise stated, C6 cells expressing N5shA were used as a representative NHE5-knockdown cell line. Human integrin $\beta 1$ ( $h I T G B 1)$ cDNA was amplified from pMD-ITGBI (\#HG10587-M; Sino Biological, Beijing, China) using the following primers: 5'-CCTCGAAAGGCCTCTGAGGCC ATGAATTTACAACCAATTTTCTGG-3' and 5'-GGAAGC TTGGCCTGACAGGCCTCATTTTCCCTCATACTTCGG $-3^{\prime}$. The PCR amplicon was then ligated into a mammalian expression vector pSBtet-Pur [18] (Addgene, Cambridge, MA, USA). Modified site-directed mutagenesis [19, 20] was employed to insert an HA-tag after the 24th amino acid residue of $h I T G B l$ using the following primers: 5'-CCATAT GACGTGCCCGACTACGCCGGAGAAAATAGATGTTTA AAAGCAAATGCC-3' (sense) and 5'-GGCGTAGTCGGG CACGTCATATGGGTATTCATCTGTTTGAGCAAACAC $-3^{\prime}$ (antisense).

\section{Semi-quantitative determination of MET and EGFR signaling}

Serum-starved cells were stimulated by adding FBS to culture media to a final concentration of $10 \%$. Cells were then washed with PBS and lysed in radioimmunoprecipitation assay (RIPA) buffer (50 mM Tris- $\mathrm{HCl}, 50 \mathrm{mM} \mathrm{NaCl}, 0.1 \%$ SDS, $0.5 \%$ sodium deoxycholate, and $1 \%$ nonidet $\mathrm{P}-40 ; \mathrm{pH}$ 7.2) supplemented with protease inhibitor (11697498001; Sigma Aldrich, St. Louis, MO) and phosphatase inhibitor
(4906845001, Sigma Aldrich). Debris-cleared lysates were mixed with SDS sample buffer (125 mM Tris-HCl, 4\% SDS, $20 \%$ glycerol, $0.004 \%$ bromophenol blue, and $10 \%$ dithiothreitol; pH 6.8) and denatured at $65^{\circ} \mathrm{C}$ for $20 \mathrm{~min}$. Equal amounts of proteins were subjected to SDS-PAGE, followed by western blotting. Antibodies against tyrosine-and/or threonine-phosphorylated EGFR (pY1068, 3777; Cell Signalling Technology, Danvers, MA), MET (pY1234/Y1235, 44-888G; Thermo Fisher, Waltham, MA), ERK1/2 (pT202/ Y204, 9101; Cell Signalling Technology), and AKT (pT308, 1308, Cell Signalling Technology) were used to detect the phosphorylation status of these signaling molecules. Blots were reprobed with antibodies against EGFR (sc-03; Santa Cruz Biotechnology, Dallas, TX), MET (3127; Cell Signalling Technology), AKT (9272, Cell Signalling Technology), ERK1/2 (9102; Cell Signalling Technology), NHE1 (611774; BD Biosciences, San Jose, CA), NHE5 (GenScript, Piscataway, NJ) $[13,16]$, and $\mathrm{Na}^{+} / \mathrm{K}^{+}$-ATPase (NKA, $\alpha 5$; Developmental Studies Hybridoma Bank, Iowa City, IA).

\section{Spheroid growth assays}

C6 spheroid cultures were generated according to the liquid overlay method, as previously described [21]. Ninety-sixwell flat-bottomed plates were coated with a 1:1 mixture of dissolved 1\% agarose and pre-warmed DMEM. After the agarose mixture had solidified, 500 cells $/ 100 \mu \mathrm{L}$ culture media were added to each well and the plates were centrifuged at $1500 \times g$ for $10 \mathrm{~min}$, followed by incubation at $37^{\circ} \mathrm{C}$ in $5 \% \mathrm{CO}_{2}$. Images of spheroids were captured with a light microscope. Spheroid growth was assessed by acid phosphatase assay (APH), as previously described [22].

\section{Determination of cell attachment}

Auguiar et al. showed that C6 cells exhibit stronger attachment and greater migration on Collagen IV than on other ECM proteins (laminin and fibronectin) [23]. This study has provided us with the rational in using collagen IV as ECM. Two-thousand cells were seeded onto collagen IV (col IV; $0.06 \mu \mathrm{g} / \mathrm{mL}$ )- or polyethyleneimine (PEI; $25 \mathrm{mM}$ )-coated 96-well plates. After the cells had been allowed to spread for various time periods, non-adherent cells were gently washed away with PBS and cell attachment was terminated by fixation in 3\% PFA supplemented with $500 \mathrm{ng} / \mathrm{mL}$ Hoechst-33342 dye (Sigma Aldrich) for $15 \mathrm{~min}$ at room temperature. Images were captured and analyzed by a Cellomics Arrayscan VTI high content screening system (Thermo Fischer Scientific) according to the protocol provided by the manufacturer. 


\section{Measurement of cell spreading areas}

Cells spread onto PEI- or col IV-coated coverslips were fixed, permeabilized, and incubated with Alexa-Fluor488-conjugated Alexa 488-phalloidin and DRAQ5 to visualize the actin cytoskeleton and nuclei, respectively. Images were captured with a Leica TCS-SP8 laser scanning confocal microscope and analyzed by Fuji Is Just ImageJ (Fuji) software. The brightness and contrast of the images were autoadjusted, followed by determination of the auto-threshold of the signal for these images to generate binary images. The area of the cells was measured using the 'measure' plugin of the software.

\section{Cell surface biotinylation and endocytosis assays}

Cell surface biotinylation and endocytosis were assessed as described previously [14] with some modifications. Subconfluent cells seeded onto fibronectin-coated plates were labeled with $0.3 \mathrm{mg} / \mathrm{mL}$ EZ-Link ${ }^{\mathrm{TM}}$ Sulfo-NHS-SSBiotin (Thermo Fisher Scientific) for $35 \mathrm{~min}$ at $4{ }^{\circ} \mathrm{C}$, followed by quenching by PBS supplemented with $20 \mathrm{mM}$ glycine. Cells were then incubated in conditioned growth medium at $37^{\circ} \mathrm{C}$ for 0 or $10 \mathrm{~min}$ to allow for internalization of the cell surface proteins. Biotin was removed from the non-internalized cell surface proteins using ice-cold membrane-impermeable cleavage buffer containing $50 \mathrm{mM}$ glutathione. Some samples were not subjected to the cleavage step ('non-cleaved proteins') to determine intrinsic degradation during incubation at $37^{\circ} \mathrm{C}$. Next, $30 \mu \mathrm{g}$ of noncleaved proteins and $160 \mu \mathrm{g}$ cleaved proteins were incubated

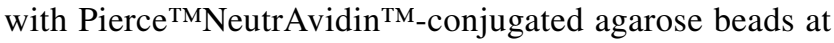
$4{ }^{\circ} \mathrm{C}$. The eluted protein samples as well as $6 \mu \mathrm{g}$ of the total protein were analyzed by western blotting. Densitometry analysis was performed using Image $\mathbf{J}$ software. The following equation was used to determine the internalization percentage: $\left[10^{\prime}(+)-0^{\prime}(+)\right] / 10^{\prime}(-)$, where $10^{\prime}(+)$ and $0^{\prime}(+)$ represent the densitometric signal of biotinylated proteins that were treated with cleavage buffer after $10 \mathrm{~min}$ and $0 \mathrm{~min}$ of post-biotinylation incubations, respectively. $10^{\prime}(-)$ represents the biotinylated proteins (not treated with cleavage buffer) after 10 min of post-biotinylation incubation, which includes both internalized and non-internalized populations.

\section{Degradation assays}

Cells were seeded overnight and cell surface proteins were biotinylated as described above. After quenching, the cells were incubated at $37{ }^{\circ} \mathrm{C}$ in a humidified atmosphere with $5 \% \mathrm{CO}_{2}$ for the indicated times, followed by rinsing twice with $1 \times$ PBS (pH 7.4) and lysing with ice-cold RIPA buffer supplemented with proteinase inhibitor cocktails. Approximately $50-60 \mu \mathrm{g}$ of total protein was incubated with Pierce
NeutrAvidin ${ }^{\mathrm{TM}}$ agarose beads overnight at $4{ }^{\circ} \mathrm{C}$ with rotation. The beads were washed three times with ice-cold $1 \%$ NP-40 in PBS (pH 7.4) and the absorbed protein was eluted with $2 \times$ Laemmli sample buffer. The eluted protein and approximately $6 \mu \mathrm{g}$ of the total protein were immunoblotted for in integrin $\beta 1$. Densitometry analysis was performed using Image $\mathbf{J}$ software. The relative pool of remaining biotinylated integrin $\beta 1$ at the indicated time was obtained by normalizing the value to that of time 0 in the respective cell lines.

\section{Recycling assays}

Recycling assays were performed as described previously [14] with some modifications. After cell surface biotinylation, chase incubation, and cleavage, cells were subjected to a second round of incubation at $37^{\circ} \mathrm{C}$ for $10 \mathrm{~min}$ to allow the internalized biotin-labeled proteins to be recycled back to the cell surface. Treatment of cells with cleavage buffer led to the removal of biotin from proteins that had been recycled to the cell surface. The cells were then rinsed several times with ice-cold PBS-CM (PBS containing $0.1 \mathrm{mM}$ of $\mathrm{CaCl}_{2}$ and $1 \mathrm{mM}$ of $\left.\mathrm{MgCl}_{2}, \mathrm{pH} 8.0\right)$ and lysed. To determine the intrinsic degradation, some samples were not subjected to the second post-incubation cleavage. Approximately $450 \mu \mathrm{g}$ of protein was incubated with Pierce NeutrAvidin ${ }^{\mathrm{TM}}$ conjugated agarose beads. The eluted protein samples as well as $\sim 6 \mu \mathrm{g}$ of the total protein that was not subjected to the NeutrAvidin ${ }^{\mathrm{TM}}$ agarose bead-pulldown were analyzed by immunoblotting. Densitometric analysis was performed using Image $\mathbf{J}$ software. The following equation was used to assess the ratio of recycled proteins after $10 \mathrm{~min}\left(10^{\prime}\right)$ of second incubation at $37^{\circ} \mathrm{C}: 1-\left(10^{\prime}(+) / 10^{\prime}(-)\right)$, in which $10^{\prime}(+)$ is the densitometric signal of sample treated with second post-incubation cleavage while $10^{\prime}(-)$ is the corresponding sample without second post-incubation cleavage.

\section{Antibody feeding assays and colocalization analysis}

HA-hITGB1 expression was induced by $200 \mathrm{ng} / \mathrm{mL}$ doxycycline and labeled by adding anti-HA antibody to the bathing solution of cells. After quick washing, cells were incubated in conditioned complete medium at $37{ }^{\circ} \mathrm{C}$ for $10 \mathrm{~min}$. Intracellular localization of HA-hITGBI and EEAl was determined by immunofluorescence microscopy. Nuclei were visualized by DRAQ5 ${ }^{\mathrm{TM}}$ (\#62254; Thermo Fisher). The images were acquired using Leica TCS-SP8 laser scanning confocal microscope equipped with $\times 63$ oil immersion objectives (NA 1.40) with a zoom factor of 2.80, Diode/ Argon/HeNe lasers, and HyDTM hybrid detectors (Wetzlar, Germany). The pixel sizes of the images were $0.129 \mu \mathrm{m}$. Confocal microscopic images were analyzed using Just Another Colocalization Plugin (JACoP) in Fiji Is Just ImageJ 
(Fiji) software to obtain the Pearson's correlation coefficient between the signals.

\section{Invasion assays}

The invasive ability of each cell line was assessed using BD BioCoatMatrigel Invasion Chambers for 24-well plates (BD Bioscience, Franklin Lakes, NJ, USA), according to the manufacturer's instructions. In brief, Matrigel on the polycarbonate membrane with $8 \mu \mathrm{m}$ pore was rehydrated using $750 \mu \mathrm{L}$ of serum-free medium, after which $750 \mu \mathrm{L}$ of fresh medium containing $10 \%$ FBS as chemoattractant was added to the lower chamber. Next, $0.5 \mathrm{~mL}$ of each cell line $\left(1 \times 10^{5}\right.$ cells $\left./ \mathrm{mL}\right)$ in serum-free medium was seeded into the upper chamber. After $24 \mathrm{~h}$, cells in the upper chamber were removed, and those that had invaded through the Matrigel were fixed in $100 \%$ methanol and stained with hematoxylin. Using three membranes per group, invading cells were counted in several fields under a microscope with a $10 \times$ objective. The following equation was used to calculate the proportion of invading cells: $\%$ invasion $=($ mean number of cells invading through Matrigel/mean number of cells migrating through Matrigel-uncoated polycarbonate membrane) $\times 100$.

\section{Mouse subcutaneous xenograft models}

Animals were treated in accordance with the Fundamental Guidelines for Proper Conduct of Animal Experiment and Related Activities in Academic Research Institutions, under the jurisdiction of the Ministry of Education, Culture, Sports, Science and Technology of Japan. All animal experiments were approved by the Committee on Animal Experimentation of Kanazawa University. Female BALB/cnu/nu mice (Charles River Laboratories, Inc., Yokohama, Japan) of 4 weeks of age were maintained in a sterile environment. NHE1-knockdown, NHE5-knockdown and control cells were cultured in DMEM with $10 \%$ FBS and appropriate selection antibiotics (G418 and hygromycin) to subconfluency, and $5 \times 10^{6}$ cells in $100 \mu \mathrm{L}$ of DMEM without FBS were subcutaneously injected into the dorsal side of each mouse. Animals were carefully monitored, and tumor volumes were measured on days $4,6,8$, and 10 . The tumor volume $(\mathrm{V})$ was calculated according to the formula $\mathrm{V}=\mathrm{AB}^{2} / 2$, where $\mathrm{A}$ is the length of the major axis, and $\mathrm{B}$ that of the minor axis. On day 10, mice were killed and tumor specimens were collected for histological and immunohistochemical examination.

\section{Histology and immunohistochemistry}

Tumor specimens were fixed in $10 \%$ neutral buffered formalin and embedded in paraffin. Sections were stained with hematoxylin and eosin (HE) for assessment of invasiveness into the surrounding tissue. Deparaffinized sections were pretreated by autoclaving in $10 \%$ citric acid buffer $(\mathrm{pH} 8.0)$ at $120{ }^{\circ} \mathrm{C}$ for $15 \mathrm{~min}$. Following treatment with protein block serum (DakoCytomation) for $10 \mathrm{~min}$ and 2\% skim milk for 30 min to block non-specific reactions, sections were incubated with the primary antibody at $4{ }^{\circ} \mathrm{C}$ overnight. EnVision polymer solution (HRP; DakoCytomation) was then applied for $1 \mathrm{~h}$. Proteins were visualized with $0.02 \%$ 3, 3'-diaminobenzidinetetrahydrochloride solution. Sections were then lightly counterstained with hematoxylin.

\section{Determination of Ki-67 scores}

Anti-Ki-67 antibodies (M7248, diluted 1:25; DakoCytomation, Kyoto, Japan) were used for immunohistochemical assessments. Slides were scanned and higher densitystained areas were located using an Olympus microscope. Three independent authors (TK, SF, and MN) manually counted at least 2000 tumor cells in three randomly chosen microscopic fields for calculating the average percentage of positive tumor cells.

\section{Results}

\section{MET and EGFR signaling are impaired in NHE5-knockdown glioma cells}

FBS stimulation of C6 glioma cells led to phosphorylation of MET, EGFR, AKT, and ERK1/2 (Fig. 1). When serumstarved NHE5-knockdown cells were stimulated with FBS, phosphorylation of MET and EGFR were barely detectable, and phosphorylation of AKT and ERK1/2 were also greatly diminished. A slight but a significant decrease in the total protein levels of MET and EGFR was also detectable in NHE5-depleted cells. NHE1 knockdown resulted in a slight reduction in the phosphorylation of AKT and ERK1/2, whereas there was no effect on MET and EGFR phosphorylation.

\section{Proliferation in 3D spheroid cultures is impaired in NHE5-knockdown glioma cells}

Spheroid cultures reflect the 3D cellular context and can serve as an excellent model to characterize the cellular behavior of tumor tissues [22]. Proliferation of NHE5knockdown spheroids was significantly slower than that of the control cells, whereas proliferation was not affected by NHE1-knockdown (Fig. 2a-c). 

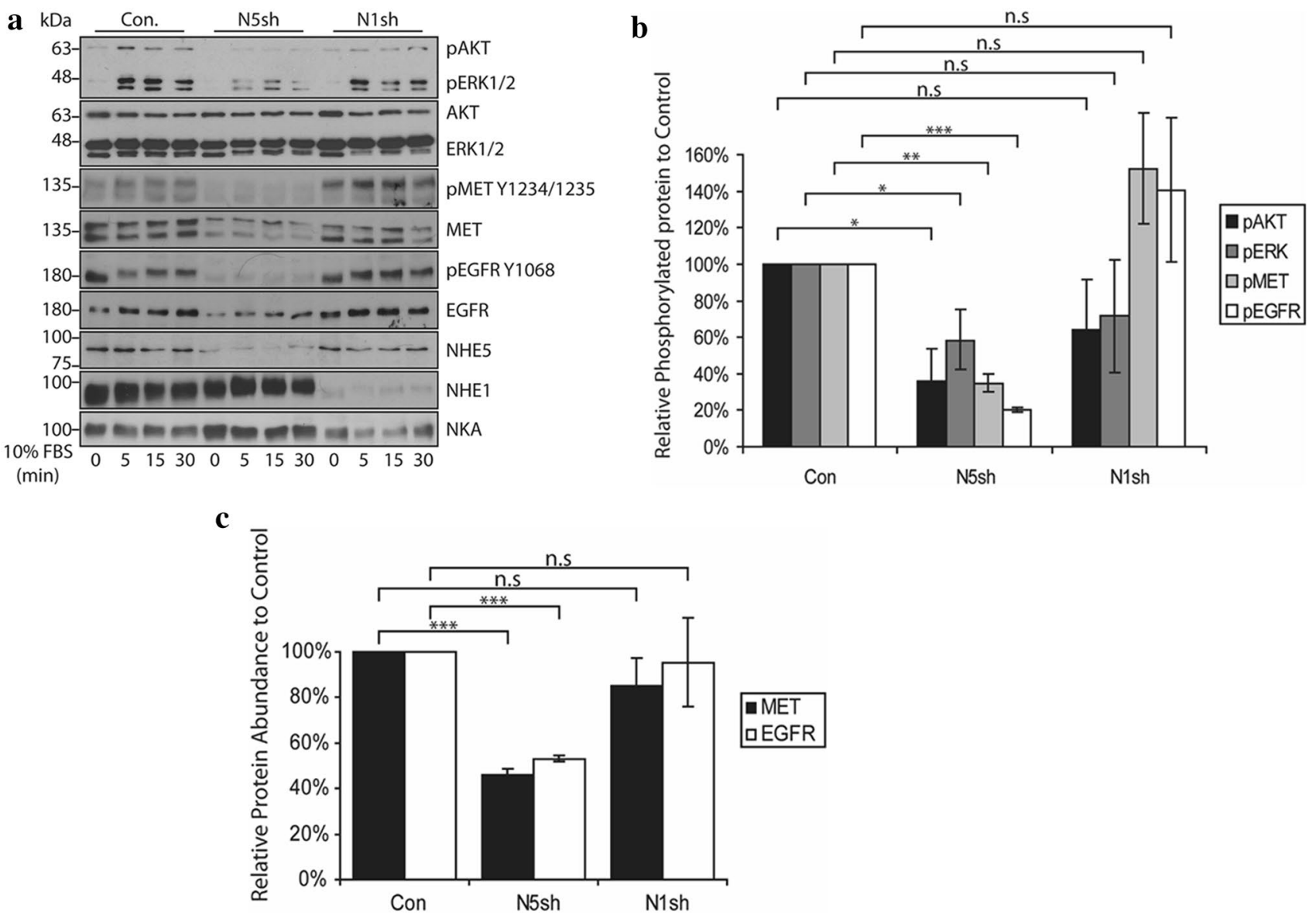

Fig. 1 NHE5 depletion dramatically downregulates phosphorylation of MET and EGFR and attenuates PI3 K and MAPK signaling. a Serum-starved C6 cells stably expressing shRNA plasmid against NHE5 (N5sh), NHE1 (N1sh), or scrambled sequence (Con) were stimulated with culture medium containing $10 \%$ FBS for 0,5 , 15 , or $30 \mathrm{~min}$ and analyzed by western blotting. Activation of AKT, ERK1/2, MET, and EGFR and was assessed by antibodies that specifically recognize the phosphorylated forms of the respective proteins. b Densitometric analysis of phosphorylated AKT, ERK1/2, MET, and EGFR at 15 min post FBS stimulation is shown. Band intensities of

\section{Spreading on collagen is impaired in NHE5-knockdown glioma cells}

Cell spreading and adhesion to extracellular matrices are important for cell survival, cell migration, and tissue remodeling [24]. To examine the capability of cells to adhere to different matrices, equal numbers of NHE5knockdown cells, NHE1-knockdown cells, and control cells were seeded onto glass coverslips coated with either PEI (non-integrin matrix) or col IV (integrin matrix). The number of NHE5-knockdown cells (N5shA, N5shB, and $\mathrm{N} 5 \mathrm{shC}$ ) attached to col IV-coated coverslips after $10 \mathrm{~min}$ of seeding was significantly smaller than that of scrambled control cells (Con) and NHE1-knockdown cells (N1sh) (Fig. 3a, b). There was no apparent difference in the phosphorylated proteins from NHE5-KD (N5sh), NHE1-KD (N1sh), or control (Con) cell lysate were normalized to that of control. Mean \pm SD of three experiments are shown. $P$ Values were obtained from Student's $t$ test. $* p<0.05, * * p<0.01, * * * p<0.001$, n.s not significant). c Densitometric analysis of total MET and EGFR protein from NHE5-KD (N5sh), NHE1-KD (N1sh), or control (Con) cells is shown. Band intensities of total MET or EGFR from respective cell lysates were normalized to that of control cell lysate. Mean \pm SD of three experiments are shown. $P$ Values were obtained from Student's $t$ test. ${ }^{*} p<0.05,{ }^{*} p<0.01, * * * p<0.001$, n.s not significant)

number of cells attached to PEI-coated coverslips among the different cell lines. We next quantitatively determined the cell spreading area of NHE5-knockdown cells, NHE1knockdown cells, and control cells using confocal microscopy. The same number of cells was seeded onto col IV- or PEI-coated coverslips and incubated at $37{ }^{\circ} \mathrm{C}$ for different time durations. Cells were then fixed, and the area to which cells were attached was calculated. Spreading areas of the three NHE5-knockdown cell groups expressing different shRNAs (N5shA, N5shB, and N5shC) were notably smaller than those of $\mathrm{NHEl-knockdown}$ cells and control cells when cells were spread over col IV-coated coverslips (Fig. 3c, e). In contrast, when cells were spread over PEIcoated coverslips, there was no apparent difference in the areas spread by the different cell lines (Fig. 3d, e). 

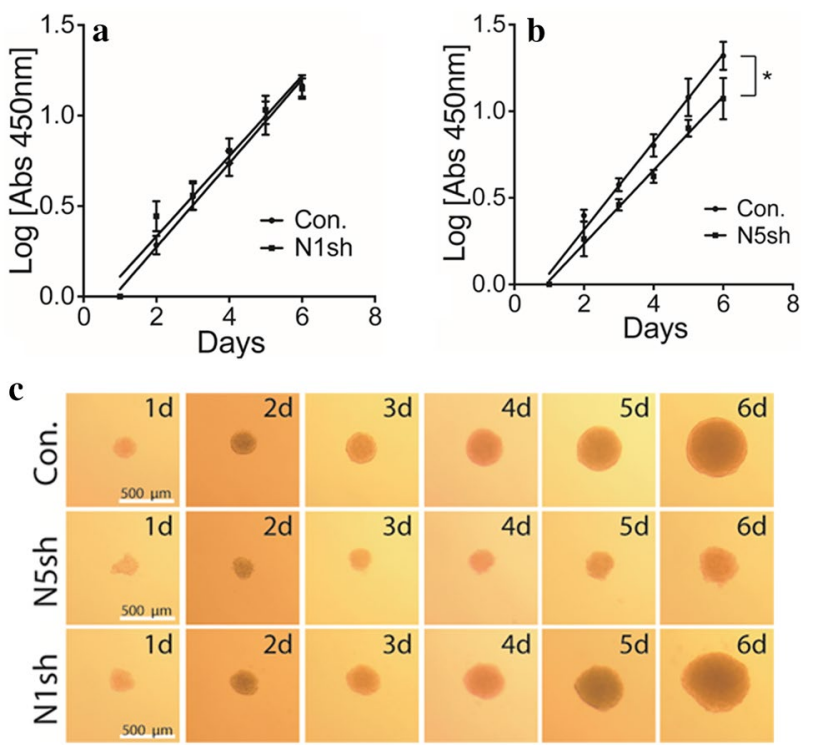

Fig. 2 Depletion of NHE5 reduces the growth of spheroid cultures. a, b Log-transformed growth curves of spheroid cultures of C6 cells stably expressing shRNA against NHE1 (N1sh), NHE5 (N5sh), and scrambled sequence (Con) over the indicated times. The growth of spheroid cultures was assessed by APH assay. Measured Absorbance at $450 \mathrm{~nm}$ of indicated time for each cell line were converted to log scale and plotted on Graphpad. Best-fitted curve of NHE5- or NHE1knockdown cells were compared to control cells by linear regression analysis, where slopes and intercepts of the resulted curves were compared. Graphs represent mean \pm SD of quadruplicate samples $\left({ }^{*} P<0.05\right)$. c Representative microscopic images of spheroid cultures (bars $=500 \mu \mathrm{m})$

\section{NHE5 knockdown diminishes endocytic recycling of integrin $\beta 1$}

Integrins are heterodimeric transmembrane receptors consisting of $\alpha$ and $\beta$ subunits and facilitate cell attachment to extracellular matrices such as collagens [25]. The $\beta 1$ subunit is abundantly expressed in C6 cells and has an important role in cell adhesion and spreading on collagen [26]. We next investigated the possible role of NHE5 in trafficking and its effect on the stability of integrin $\beta 1$. The abundance of integrin $\beta 1$ in the plasma membrane of NHE5-knockdown cells was significantly lower than that in control cells (Fig. 4a). To examine the effect of NHE5 knockdown on endocytic trafficking of integrin $\beta 1$, we performed cell surface biotinylation and endocytosis assays. In these experiments, the surface of the cells was biotinylated followed by chase incubation to facilitate endocytosis of the biotinlabeled membrane proteins. Cells were then treated with a membrane-impermeable reducing agent that selectively removes biotin from non-internalized proteins present on the plasma membrane. Treatment of NHE5-knockdown cells and control cells with the reducing agent resulted in almost complete disappearance of biotinylated integrin $\beta 1$ (Fig. 4a). When cells were subjected to post-biotinylation chase incubation, the internalized integrin $\beta 1$ population that was not accessible to the cleavage buffer became detectable by western blotting. Interestingly, the intensity of integrin $\beta 1$ was substantially stronger in NHE5-knockdown cells than in the control cells, suggesting that there is a larger population of internalized integrin $\beta 1$ in NHE5-knockdown cells (Fig. 4a, b). To further investigate this possibility, NHE5-knockdown cells and $\mathrm{C} 6$ cells stably expressing N-terminally HA-tagged human integrin $\beta 1$ (HA-hITGB1) driven by the tetracyclineinducible promoter were established. Based on the resolved 3D structure, we designed the position of the HA-tag so that it was exposed to the extracellular space of integrin $\beta 1$ [27]. NHE5-knockdown cells and control cells stably expressing inducible $H A$-hITGBI were cultured overnight at $37{ }^{\circ} \mathrm{C}$ in complete medium containing $200 \mathrm{ng} / \mathrm{mL}$ of doxycycline, and HA-hITGB 1 protein residing in the plasma membrane was labeled in cell at $4{ }^{\circ} \mathrm{C}$ by adding anti-HA antibody to the bathing solution. After extensive washing and quenching, cells were subjected to incubation at $37{ }^{\circ} \mathrm{C}$ to render cell surface receptors subject to endocytosis. The cells were then fixed and the intracellular localization of HAhITGB1 was determined by immunofluorescence microscopy. Endocytosed integrin $\beta 1$ exhibited a higher degree of colocalization with an early endosomal marker EEA1 in NHE5-knockdown cells than in control cells (Fig. 4c, d). As decreased endocytic recycling may increase the endosomal population of receptor proteins, we next examined endocytic recycling. Cell surface biotinylated proteins were internalized, and the non-internalized population was removed by treating cells with cleavage buffer containing a membraneimpermeable reducing agent. The cells were then subjected to a second round of chase incubation to facilitate recycling from endosomes to the plasma membrane, followed by the treatment with cleavage buffer ('second cleavage'; Fig. 4e) to eliminate biotin from proteins that have returned to the plasma membrane. Finally, biotinylated proteins were affinity-purified and integrin $\beta 1$ was detected by western blotting. The signal intensity of biotinylated integrin $\beta 1$ that represents the non-recycled population was higher in NHE5knockdown cells than in the control cells, indicating that integrin $\beta 1$ recycling is impaired in NHE5-knockdown cells (Fig. 4e, f).

\section{NHE5-knockdown accelerates degradation of integrin $\beta 1$}

Internalized receptors can be either recycled back to the plasma membrane or targeted to lysosomes for degradation. It was previously shown that degradation of integrin $\beta 1$ is accelerated when its recycling is perturbed [28], which led us to hypothesize that NHE5 influences 

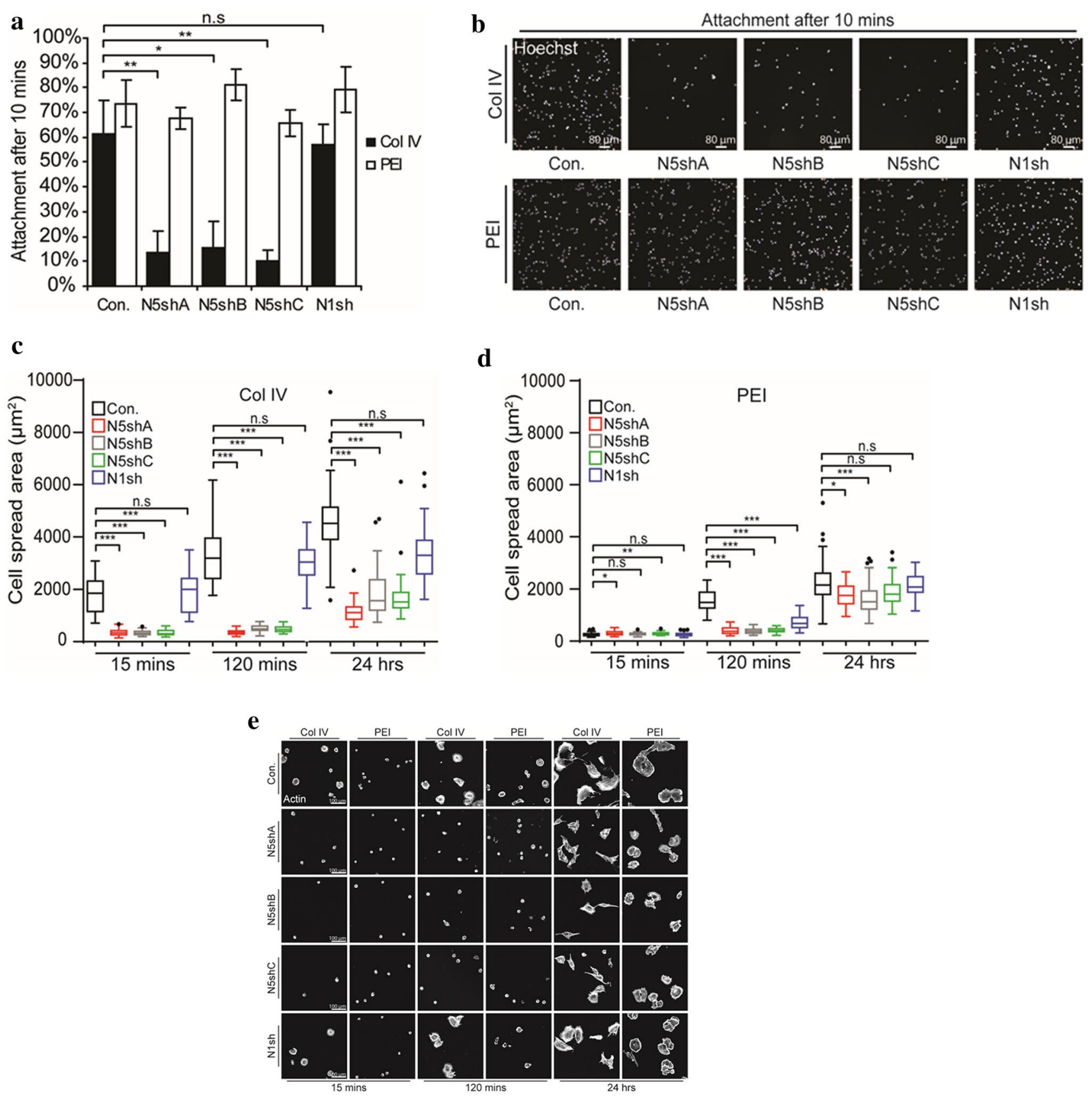

Fig. 3 NHE5 knockdown impedes cell attachment and spreading. a C6 cells stably expressing shRNA against NHE5 (N5shA, B, C), NHE1 (N1sh), or scrambled control (Con) were subjected to cell attachment assays on collagen IV (col IV)- or polyethyleneimine (PEI)-coated surfaces. Data are presented as percentages of cells attached to different substrates after $10 \mathrm{~min}$ of spreading. Means and standard deviations are plotted $(* P<0.05 ; * * P<0.01 ; n . s$ not significant; $\mathrm{n}=3$ ) bar $=80 \mu \mathrm{m}$. b Representative images of control, NHE5-knockdown, and NHE1-knockdown cells spread onto a col

degradation of integrin $\beta 1$. Integrin $\beta 1$ was pulse-labeled by cell surface biotinylation and the degradation rate was determined after chase incubation for different durations.
IV- or PEI-coated plate for $10 \mathrm{~min}$. c, $\mathbf{d}$ The spread area of randomly selected cells was analyzed $(n>40)$ and the distribution is shown as box-and-whisker plots with the ends of the whiskers set at $1.5 \times$ interquartile range (IQR) above the third quartile and $1.5 \times \mathrm{IQR}$ below the first quartile. Kruskal-Wallis tests with Dunn's tests with Bonferroni correction for pair-wise comparison were performed. $\left({ }^{*} P<0.05\right.$; $* * P<0.01 ; * * * P<0.001 ;$ n.s $=$ not significant). e Representative confocal images of control, NHE5-, or NHE1-knockdown cells on col IVor PEI-coated surfaces after $15 \mathrm{~min}$. Bar $=100 \mu \mathrm{m}$

The half-life of integrin $\beta 1$ in control cells was approximately $18 \mathrm{~h}$ whereas that of NHE5-knockdown cells was approximately $4 \mathrm{~h}$ (Fig. $4 \mathrm{~g}, \mathrm{~h}$ ). 


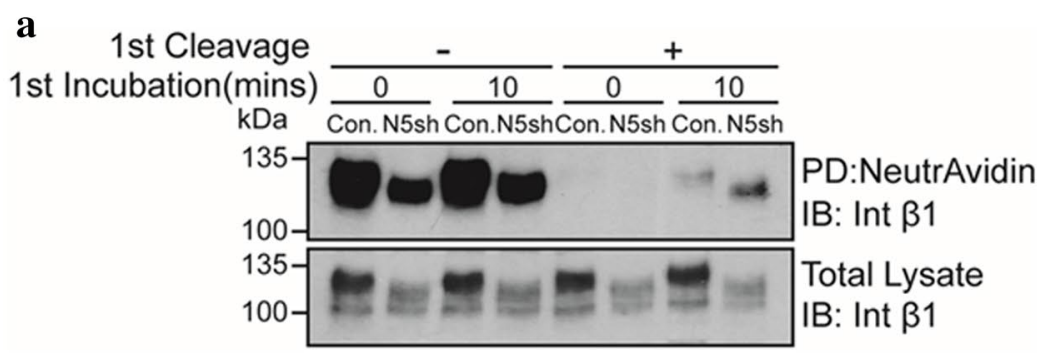

b

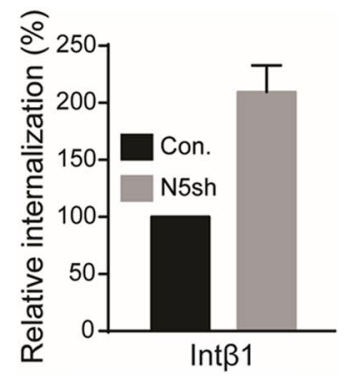

c

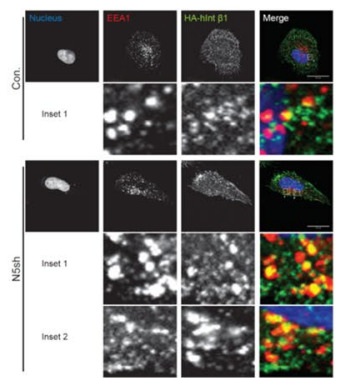

e

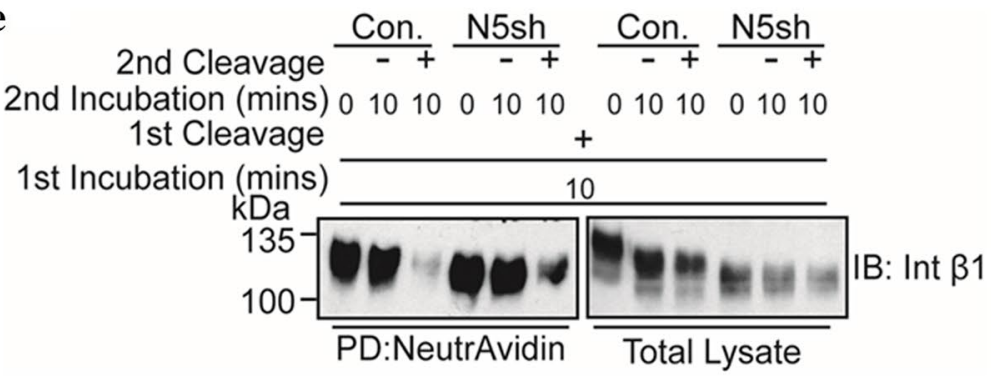

g

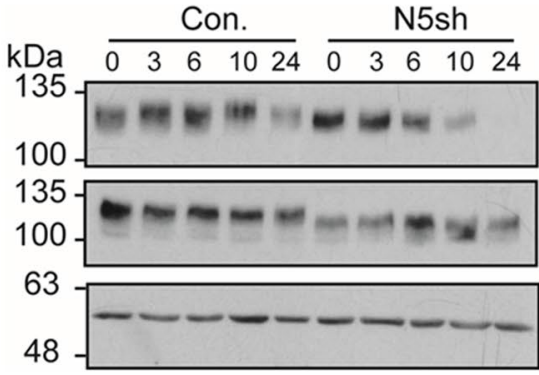

Chase (hrs)

PD:NeutrAvidin

IB: Int $\beta 1$

Lysate

IB: Int $\beta 1$

Lysate

IB: $\beta$-tub
Fig. 4 NHE5 regulates intracellular trafficking of integrin $\beta 1$. a Representative data of cell surface expression and internalization of integrin $\beta 1$. b Relative internalization of integrin $\beta 1$ in NHE5knockdown cells and control cells. Means and standard deviations are shown $(n=3)$. c Representative confocal images of the antibody feeding assays. Localization of internalized HA-tagged integrin $\beta 1$ (HA-hITGB1, green) in NHE5-knockdown cells and control cells was analyzed by immunofluorescence microscopy. Early endosomes and nuclei are marked by anti-EEA1 antibody (red) and Hoechst dye (blue), respectively. Bars $=20 \mu \mathrm{m}$. The zoomed insets (white squares) that show the colocalization between EEA1 and HA-hITGB1. d Scatter plot of Pearson's correlation coefficient of colocalization between
HA-hITGB1 and EEA1 in control and NHE5-knockdown cells. The median and interquartile range are shown $(* * * P<0.001$; MannWhitney test; $n=2$ ). e Representative result of integrin $\beta 1$ recycling assays. Recycling of cell surface biotinylated and internalized proteins was determined. Following the 'second cleavage', only nonrecycled integrin $\beta 1$ that was protected from cleavage was detected. f Relative recycling was calculated by normalizing the recycled pool of NHE5-knockdown cells to that of control cells. Means and standard deviations are shown $(n=3)$. g Representative result of integrin $\beta 1$ degradation assay. $\mathbf{h}$ Relative degradation of integrin $\beta 1$ in control or NHE5-knockdown cells plotted over time. Mean \pm SD from three independent experiments are shown 


\section{NHE5 is required for cell invasion through Matrigel}

Endocytosis and recycling of integrins are increasingly recognized as crucial regulatory events of cell migration and invasion by regulating the balance between cell-surface availability and degradation of membrane proteins, which led us to examine invasion capability of NHE5-knockdown cells [29]. Invasion capability across Matrigel was substantially slower in NHE5-knockdown cells compared with NHE1-knockdown cells and control cells (Fig. 5). Because BD Matrigel contained some growth factors, such as TGFbeta, EGF and IGF etc., our invasion assay cannot be entirely distinguished from chemotaxis assay. However, these results suggest that NHE5 plays an important role in invasion through matrices.

\section{NHE5-knockdown reduces the rate of xenograft tumor growth}

The abovementioned results have indicated the possible involvement of NHE5 in tumor growth and progression. We next examined the growth of tumors derived from NHE5knockdown cells, NHE1-knockdown cells, and control cells in vivo. The volume of NHE5-knockdown (NHE5-KD) tumors was markedly smaller than that of tumors originating from parental $\mathrm{C} 6$ cells (C6 wt) and C6 cells expressing control vector (CV) (Fig. 6). The size of NHEl-knockdown tumors (NHE1-KD) was slightly smaller than C6 wt or CV tumors, but significantly larger than that of NHE5-KD tumors. Histological characterization revealed that $\mathrm{C} 6 \mathrm{wt}$

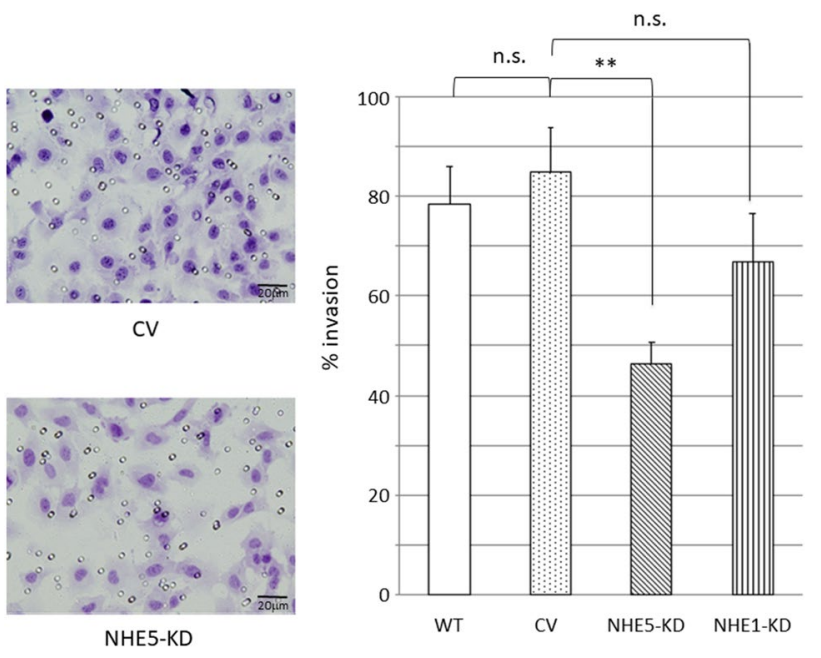

Fig. 5 Invasion assays. (left) Representative images of invaded C6 cells expressing control vector (upper; CV) and NHE5-knockdown cells (lower; NHE5-KD). (right) Invaded cells in each clone were counted. (**P<0.01; n.s. not significant). WT, wild type NHE; CV, control vector transfection; NHE5-KD, NHE5-knockdown; NHE1$\mathrm{KD}$, NHE1-knockdown

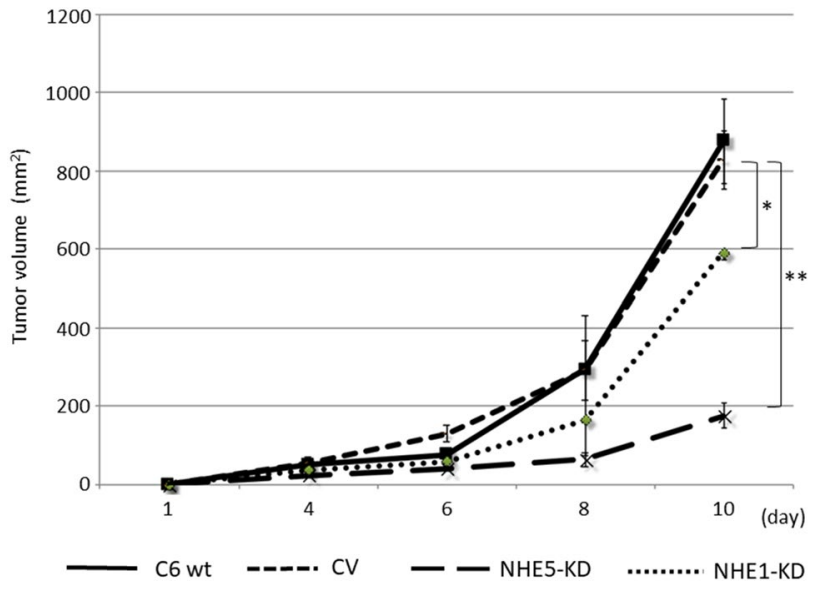

Fig. 6 Tumors derived from NHE knockdown cells were significantly smaller than those from control vector. $(* P<0.05 ; * * P<0.01)$

cells and CV cells showed severe invasion into the surrounding tissue whereas invasion of NHE5-knockdown cells to the surrounding tissue was not apparent (Fig. 7). The Ki-67 labeling index was significantly lower in NHE5-KD tumors than in CV tumors (Fig. 8).

\section{Discussion}

C6 cells express both NHE5 and NHE1, which makes them a unique model in which to investigate the role of NHE5 and NHE1 in tumor growth, invasion, and signaling. Using C6 glioma cell lines stably expressing plasmid-based shRNA as a model, we demonstrated that NHE5-knockdown greatly inhibits tumor growth. Although subcutaneous tissue is different from actual environment in the brain, it is useful to assess the malignant potential, such as proliferation and invasion as an in vivo model. The microscopic appearance of the tumor migration front, the Ki-67 labeling index of tumor xenografts, invasion assays through Matrigel, and spheroid growth experiments all suggest that $N H E 5$-knockdown cells are less invasive. NHE5-depletion downregulates the signaling pathways mediated by MET, EGFR, and integrins, whereas the influence of NHE1-depletion on these signaling pathways was limited. Amplification of EGFR is the most commonly observed genetic abnormality in glioma, and more than $50 \%$ of glioma cases are said to be associated with amplification of EGFR [30]. However, EGFR-targeted therapies have been largely ineffective for glioma due to the rapid development of drug resistance [31]. Multiple growth factor receptors including MET and EGFR are known to be overexpressed in aggressive gliomas [31-34]. As different growth factor receptors share common downstream signaling pathways, and inhibition of one growth factor receptor may upregulate other growth factor receptors, blockade of a 
Fig. 7 Histological examination by hematoxylin and eosin staining. WT, wild type; CV, control vector; NHE5-KD,

NHE5-knockdown; NHE1-KD, NHE1-knockdown. (Magnification, $\times 40$ )
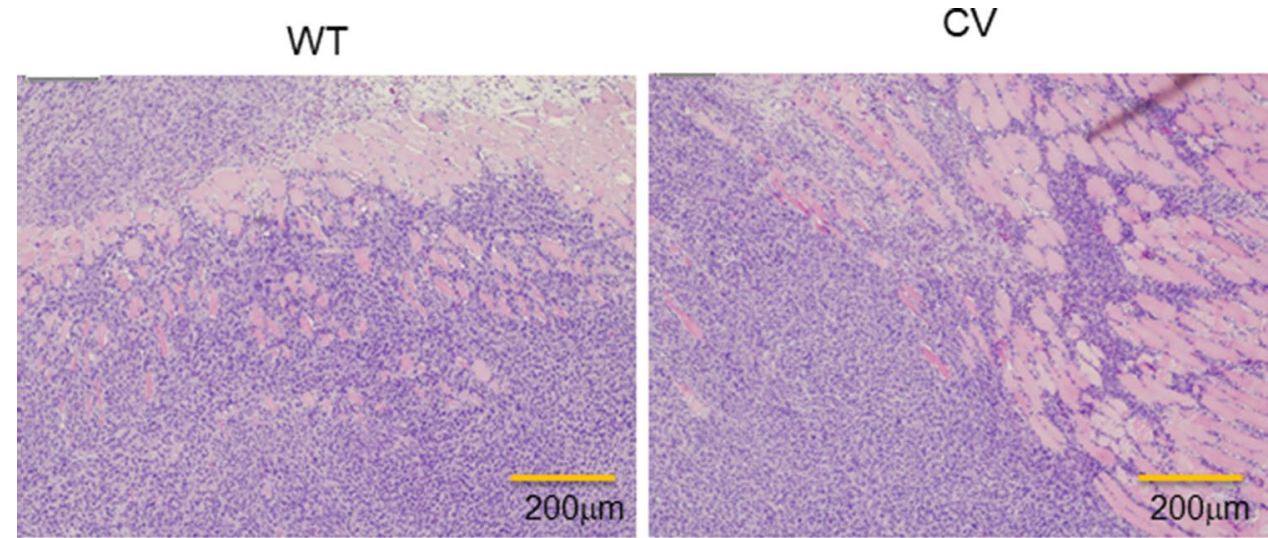

NHE5-KD

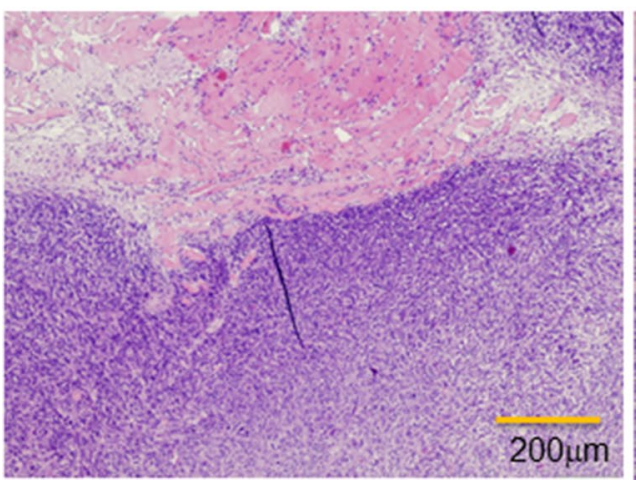

NHE1-KD

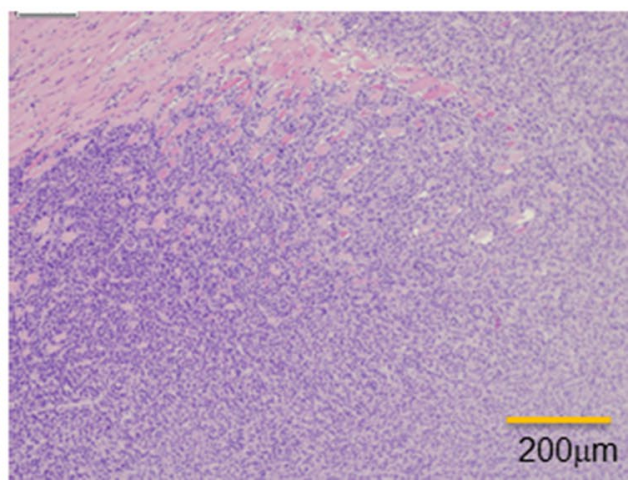

common upstream regulatory mechanism of growth factor receptors has potential as a potent anti-cancer therapy and may enable resistance against anti-EGFR therapy to be overcome $[35,36]$. In this respect, our current findings indicate NHE5 as a potential therapeutic target for glioma.
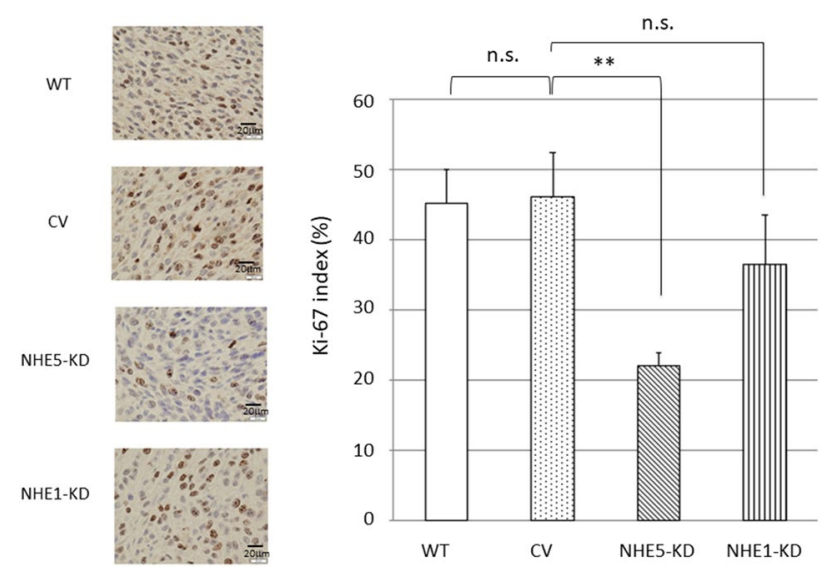

Fig. 8 Ki-67 labeling index. (left) Representative photomicrograph of Ki-67-labeled cells in each tumor. (right) Ki-67 labeling index of each tumor. (**P<0.01; n.s. not significant). WT, wild type NHE; $\mathrm{CV}$, control vector transfection: NHE5-KD, NHE5-knockdown; NHE1-KD, NHE1-knockdown
Defective attachment to collagen is a unique phenotype found in NHE5-depleted glioma cells but not in NHE1depleted cells. We have shown that NHE5 has a critical role in endocytic recycling and degradation of integrin $\beta 1$, which likely accounts, at least in part, for the defective adhesion of NHE5-knockdown glioma cells. Coordinated recyclings of MET, EGFR, and other RTKs with Integrins through Rab11FIP1 have been suggested to play a pivotal role in promoting cancer invasion [37, 38]. In addition, EGFR signaling has been implicated in trafficking of Integrin $\alpha 5 \beta 1$ to the leading edge of migrating cells [37]. NHE5 potently acidifies the lumen of recycling endosomes and facilitates the return of MET and TRKA to the plasma membrane $[14,16]$. However, it is not known whether NHE5 influences intracellular targeting and degradation of membrane receptors other than growth factor receptors. Our current findings suggest that NHE5 impacts on the function of a broader range of membrane receptors including growth factor receptors and integrins by balancing the recycling and degradation of internalized receptors.

Amiloride derivatives such as 5-( $N$-ethyl- $N$-isopropyl) amiloride (EIPA) and 5-( $N, N$-hexamethylene)-amiloride (HMA) are known to be NHE1-specific inhibitors with inhibition constants of $15 \mathrm{nM}$ and $13 \mathrm{nM}$, respectively [39, 40]. These NHE1 inhibitors are also able to inhibit NHE5; however, approximately 30 -fold higher doses are required 
to sufficiently inhibit heterologously expressed NHE5 in rodent fibroblasts [39]. It is possible that an even greater concentration of these inhibitors is required to block endogenous NHE5 activity in recycling endosomes. Thus, high concentrations may cause off-target effects by inhibiting $\mathrm{Na}^{+}$-channels and $\mathrm{Na}^{+} / \mathrm{Ca}^{2+}$-ATPases [40]. Stock and colleagues reported that losartan, an angiotensin II type 1 receptor antagonist, inhibits NHE1 activity and migration, but unfortunately increased adhesion and invasion in human melanoma (MV3) cells [8]. On the other hand, a previous screen of chemical derivatives of EIPA on $\mathrm{Na}^{+} / \mathrm{H}^{+}$ exchanger-deficient fibroblasts that heterologously express NHE3 led to the identification of NHE3-specific inhibitors. As NHE3 and NHE5 share similar pharmacological and physiological transporter properties, and the transporter activity of NHE 3 and NHE5 can be determined in a similar assay [15], it is possible that an analogous drug screening strategy used to isolate NHE3 inhibitors may identify novel NHE5 inhibitors.

Although NHE1 inhibitors are known to effectively retard cell proliferation of different malignant tumor cells [8-11], we have shown that NHE5 knockdown by shRNA markedly diminishes the proliferation and invasion of C6 glioma cells, and demonstrate that NHE5 knockdown has a significantly different effect on tumor growth and invasion in mouse xenografts compared to the impact caused by NHE1 knockdown. We previously showed that some, but not all, phenotypes of NHE5-knockdown cells are rescued by reexpression of shRNA-resistant NHE5 (functional NHE5 that has a mismatch sequence against the shRNA target region). In particular, we found that phenotypes related to intracellular targeting of MET and growth factor signaling tend not to be rescued $[13,14]$. In this regard, it is interesting to note that re-expression of shRNA-resistant NHE5 did not rescue the slow tumor growth caused by NHE5 depletion (data not shown). We postulate that physiological NHE5 expression levels within a narrow range allows the right balance between endocytic recycling and degradation of cell surface receptors including EGFR, MET, and integrins, thereby tightly regulating any signaling through these receptors.

\section{Conclusion}

Our current study presents a new hypothesis wherein neuron-type NHE5 ectopically expressed in C6 glioma cells plays a critical role in MET, EGFR, and integrin signaling in glioma. If NHE5 expression is restricted to glioma lesions, NHE5 may add diagnostic and therapeutic value. In future studies, it is important to investigate whether NHE5 expression is upregulated in pathological samples obtained from glioma patients, and identify whether there are any correlations between NHE5 expression and pathological type, prognosis, and other glioma-associated factors.

Acknowledgements We appreciate technical assistance from Dr. Yuka Numata who assisted with cell lines. We also thank H. Nikki March, $\mathrm{PhD}$, from Edanz Group (www.edanzediting.com/ac) for editing a draft of this manuscript.

Funding The authors declare that this study was not funded.

\section{Compliance with ethical standards}

Conflict of interest The authors declare that they have no conflict of interest.

Open Access This article is distributed under the terms of the Creative Commons Attribution 4.0 International License (http://creativeco mmons.org/licenses/by/4.0/), which permits unrestricted use, distribution, and reproduction in any medium, provided you give appropriate credit to the original author(s) and the source, provide a link to the Creative Commons license, and indicate if changes were made.

\section{References}

1. Gatenby RA, Gillies RJ (2008) A microenvironmental model of carcinogenesis. Nat Rev Cancer 8:56-61

2. Webb BA, Chimenti M, Jacobson MP, Barber DL (2011) Dysregulated pH: a perfect storm for cancer progression. Nat Rev Cancer 11:671-677

3. Amith SR, Fliegel L (2013) Regulation of the $\mathrm{Na}^{+} / \mathrm{H}^{+}$exchanger (NHE1) in breast cancer metastasis. Cancer Res 73:1259-1264

4. Sardet C, Franchi A, Pouyssegur J (1989) Molecular cloning, primary structure, and expression of the human growth factoractivatable $\mathrm{Na}^{+} / \mathrm{H}^{+}$antiporter. Cell 56:271-280

5. Fuster DG, Alexander RT (2014) Traditional and emerging roles for the SLC9 $\mathrm{Na}^{+} / \mathrm{H}^{+}$exchangers. Pflügers Arch 466:1-16

6. Donowitz M, Ming Tse C, Fuster D (2013) SLC9/NHE gene family, a plasma membrane and organellar family of $\mathrm{Na}^{+} / \mathrm{H}^{+}$exchangers. Mol Aspects Med 34:236-251

7. Koch A, Schwab A (2019) Cutaneous pH landscape as a facilitator of melanoma initiation and progression. Acta Physiol 225:e13105

8. Olschewski DN, Hofschröer V, Nielsen N, Seidler DG, Schwab A, Stock C (2018) The angiotensin II type 1 receptor antagonist losartan affects NHE1-dependent melanoma cell behavior. Cell Physiol Biochem 45:2560-2576

9. Andersen AP, Samsøe-Petersen J, Oernbo EK, Boedtkjer E, Moreira JMA, Kveiborg M, Pedersen SF (2018) The net acid extruders NHE1, NBCn1 and MCT4 promote mammary tumor growth through distinct but overlapping mechanisms. Int J Cancer 142:2529-2542

10. Amith SR, Fong S, Baksh S, Fliegel L (2015) $\mathrm{Na}(+) / \mathrm{H}(+)$ exchange in the tumour microenvironment: dose NHE1 drive breast cancer carcinogenesis? Int J Dev Biol 59:367-377

11. Baird NR, Orlowski J, Szabo EZ, Zaun HC, Schultheis PJ, Menon AG et al (1999) Molecular cloning, genomic organization, and functional expression of $\mathrm{Na}^{+} / \mathrm{H}^{+}$exchanger isoform 5 (NHE5) from human brain. J Biol Chem 274:4377-4382

12. Attaphitaya S, Park K, Melvin JE (1999) Molecular cloning and functional expression of a rat $\mathrm{Na}^{+} / \mathrm{H}^{+}$exchanger (NHE5) highly expressed in brain. J Biol Chem 274:4383-4388 
13. Diering GH, Mills F, Bamji SX, Numata M (2011) Regulation of dendritic spine growth through activity-dependent recruitment of the brain-enriched $\mathrm{Na}^{+} / \mathrm{H}^{+}$exchanger NHE5. Mol Biol Cell 22:2246-2257

14. Fan SH-Y, Numata Y, Numata M (2016) Endosomal $\mathrm{Na}^{+} / \mathrm{H}^{+}$ exchanger NHE5 influences MET recycling and cell migration. Mol Biol Cell 27:702-715

15. Orlowski J, Grinstein S (2011) $\mathrm{Na}^{+} / \mathrm{H}^{+}$exchangers. Compr Physiol 1:2083-2100

16. Diering GH, Numata $\mathrm{M}$ (2014) Endosomal pH in neuronal signaling and synaptic transmission: role of $\mathrm{Na} / \mathrm{H}$ exchanger NHE5. Front Physiol. https://doi.org/10.3389/fphys.2013.00412

17. Diering GH, Numata Y, Fan S, Church J, Numata M (2013) Endosomal acidification by $\mathrm{Na}^{+} / \mathrm{H}^{+}$exchanger NHE5 regulates TrkA cell-surface targeting and NGF-induced PI3 K signaling. Mol Biol Cell 24:3435-3448

18. Kowarz E, Löscher D, Marschalek R (2015) Optimized sleeping beauty transposons rapidly generate stable transgenic cell lines. Biotechnol J 10:647-653

19. Klock HE, Lesley SA (2009) The polymerase incomplete primer extension (PIPE) method applied to high-throughput cloning and site-directed mutagenesis. In: Doyle SA (ed) High throughput protein expression and purification. Switzerland, Springer, pp 91-103

20. Liu H, Naismith JH (2008) An efficient one-step site-directed deletion, insertion, single and multiple-site plasmid mutagenesis protocol. BMC Biotechnol. https://doi.org/10.1186/1472-6750-8-91

21. Carlsson J, Yuhas J (1984) Liquid-overlay culture of cellular spheroids. In: Acker H (ed) Spheroids in cancer research. Springer, Berlin, pp 1-23

22. Friedrich J, Seidel C, Ebner R, Kunz-Schughart LA (2009) Spheroid-based drug screen: considerations and practical approach. Nat Protoc 4:309-324

23. Aguiar CB, Lobao-Soares B, Alverz-Silva M, Trentin AG (2005) Glycosaminoglycans modulate C6 glioma cell adhesion to extracellular matrix components and alter proliferation and cell migration. BMC Cell Biol 6:31

24. Gumbiner BM (1996) Cell adhesion: the molecular basis of tissue architecture and morphogenesis. Cell 84:345-357

25. Hynes RO (1992) Integrins: versatility, modulation, and signaling in cell adhesion. Cell 69:11-25

26. Grobben B, De Deyn P, Slegers H (2002) Rat C6 glioma as experimental model system for the study of glioblastoma growth and invasion. Cell Tissue Res 310:257-270

27. Nagae M, Re S, Mihara E, Nogi T, Sugita Y, Takagi J (2012) Crystal structure of $\alpha 5 \beta 1$ integrin ectodomain: atomic details of the fibronectin receptor. J Cell Biol 197:131-140

28. Böttcher RT, Stremmel C, Meves A, Meyer H, Widmaier M, Tseng HY et al (2012) Sorting nexin 17 prevents lysosomal degradation of $\beta 1$ integrins by binding to the $\beta 1$-integrin tail. Nat Cell Biol 14:584-592

29. Paul NR, Jacquemet G, Caswell PT (2015) Endocytic trafficking of integrins in cell migration. Curr Biol 25:R1092-R1105

30. Furnari FB, Fenton T, Bachoo RM, Mukasa A, Stommel JM, Stegh A et al (2007) Malignant astrocytic glioma: genetics, biology, and paths to treatment. Genes Dev 21:2683-2710

31. Dunn GP, Rinne ML, Wykosky J, Genovese G, Quayle SN, Dunn IF et al (2012) Emerging insights into the molecular and cellular basis of glioblastoma. Genes Dev 26:756-784

32. Sturm D, Bender S, Jones DT, Lichter P, Grill J, Becher O et al (2014) Paediatric and adult glioblastoma: multiform (epi) genomic culprits emerge. Nat Rev Cancer 14:92-107

33. Ceccarelli M, Barthel FP, Malta TM, Sabedot TS, Salama SR, Murray BA et al (2016) Molecular profiling reveals biologically discrete subsets and pathways of progression in diffuse glioma. Cell 164:550-563

34. Beroukhim R, Getz G, Nghiemphu L, Barretina J, Hsueh T, Linhart $\mathrm{D}$ et al (2007) Assessing the significance of chromosomal aberrations in cancer: methodology and application to glioma. Proc Natl Acad Sci USA 104:20007-20012

35. Huang PH, Mukasa A, Bonavia R, Flynn RA, Brewer ZE, Cavenee WK et al (2007) Quantitative analysis of EGFRvIII cellular signaling networks reveals a combinatorial therapeutic strategy for glioblastoma. Proc Natl Acad Sci USA 104:12867-12872

36. Karamouzis MV, Konstantinopoulos PA, Papavassiliou AG (2009) Targeting MET as a strategy to overcome crosstalk-related resistance to EGFR inhibitors. Lancet Oncol 10:709-717

37. Caswell PC, Chan M, Lindsay AJ, McCaffrey MW, Boettiger D et al (2008) Rab-coupling protein coordinates recycling of $\alpha 5 \beta 1$ integrin and EGFR1 to promote cell migration in 3D microenvironments. J Cell Biol 183:143-155

38. Muller PAJ, Trinidad AG, Timpson P, Morton JP, Zanivan S, van den Berghe PVE et al (2013) Mutant p53 enhances MET trafficking and signaling to drive cell scattering and invasion. Oncogene 32:1252-1265

39. Szabo EZ, Numata M, Shull GE, Orlowski J (2000) Kinetic and pharmacological properties of human brain $\mathrm{Na}^{+} / \mathrm{H}^{+}$exchanger isoform 5 stably expressed in Chinese hamster ovary cells. J Biol Chem 275:6302-6307

40. Masereel B, Pochet L, Laeckmann D (2003) An overview of inhibitors of $\mathrm{Na}^{+} / \mathrm{H}^{+}$exchanger. Eur J Med Chem 38:547-554

Publisher's Note Springer Nature remains neutral with regard to jurisdictional claims in published maps and institutional affiliations. 